\title{
Electron Correlation Effects on the Longitudinal Polarizabilities and Second Hyperpolarizabilities of Polyenes: A Finite Field Study
}

\author{
Qingxu Li, ${ }^{1}$ Xianju Zhou, ${ }^{1}$ and Shiwei Yin ${ }^{2}$ \\ ${ }^{1}$ College of Sciences, Chongqing University of Posts and Telecommunications, Chongqing 400065, China \\ ${ }^{2}$ School of Chemistry \& Chemical Engineering, Shaanxi Normal University, Xian 710062, China \\ Correspondence should be addressed to Qingxu Li; liqx@cqupt.edu.cn
}

Received 16 April 2014; Revised 11 June 2014; Accepted 11 July 2014; Published 23 July 2014

Academic Editor: Yusheng Dou

Copyright (C) 2014 Qingxu Li et al. This is an open access article distributed under the Creative Commons Attribution License, which permits unrestricted use, distribution, and reproduction in any medium, provided the original work is properly cited.

We carry out ab initio calculations, based on finite-field scheme, of the longitudinal polarizabilities $\left(\alpha_{L}\right)$ and second hyperpolarizabilities $\left(\gamma_{L}\right)$ of conjugated polyenes, and study the effects of electron correlation, with second-order Moller-Plesset perturbation theory and coupled cluster with singles and doubles method. Calculations with density functional theory are also made to compare with wave-function based methods. Our study shows that electron correlation reduces linear longitudinal polarizability and enhances longitudinal second hyperpolarizability for short polyenes, but the effects decrease as the chain increases; choosing appropriate basis sets is important when quantitative results are required.

\section{Introduction}

The linear and nonlinear optical properties of conjugated polymers have been intensely investigated in recent decades due to their great potentials in industry. The polarizability and second hyperpolarizability of linear polyenes, the simplest conjugated polymer, have attracted research interests of many scientists. Ab initio calculations of linear $\alpha_{L}$ and $\gamma_{L}$ are severely restricted to short polyenes due to the formidable computational costs. Hurst et al. reported ab initio coupledperturbed $\mathrm{HF}$ (CPHF) calculations up to $\mathrm{C}_{22} \mathrm{H}_{24}$ more than 20 years ago [1]. Since then, researchers have showed that electron correlation effects play a key role in correctly describing the linear and nonlinear optical properties of conjugated systems [2-21]. And thus, proper treatment of electron correlation in electronic structure calculations is very important. However, accurate correlated electronic structure calculations for long polyenes require immense computational resources and thus are now impractical.

DFT method becomes more popular in recent years for its excellent balance between the accuracy and computational costs $[22,23]$. Unfortunately, researches showed that DFT with commonly used xc-functionals always fails to offer a reasonable description of nonlinear optical properties for conjugated polymers, including polyenes [12-15]. In a recent work, Sekino et al. reported their efforts on developing new xc-functional to overcome this problem [15]. They also reported their calculations based on by MP2 (second-order Moller-Plesset perturbation theory) and CCSD (coupled cluster with singles and doubles method). Eventually, MP2 is the only practical choice for long polyenes till now. CCSD is more expensive and thus more restricted when used for evaluation of $\alpha_{L}$ and $\gamma_{L}$, even for the 6-31G basis set, the smallest basis set of practical use. Champagne et al. used MP2 method to invalidate DFT in evaluating $\alpha_{L}$ and $\gamma_{L}$ of conjugated chains [12].

We found recently that, based on the semiempirical Pariser-Parr-Pople (PPP) model, CCSD predicts electron correlation results in a reduction on $\gamma_{L}$ for long polyenes, while MP2 predicts electron correlation always enhances $\gamma_{L}$ and thus gives wrong predictions for electron correlation contributions to the $\gamma_{L}$ of long polyenes [9]. The primary ab initio CCSD calculations we made with 6-31G basis set lead to same conclusions to semiempirical PPP model. 
Hurst et al. showed that basis sets of quasi-double zeta quality are good enough, particularly for the longitudinal component. Since their conclusion were based on CPHF calculations, we wonder if more reliable basis sets will still support this point. Calculations with various basis sets are thus performed and reported in this paper. Results obtained by three commonly-used DFT schemes are also presented for the sake of completeness.

\section{Computational Details}

The geometries of polyenes used in this study were taken from [12] (Figure 1(a) in [12]). Electronic energies for the system in different electric fields are calculated with Gaussian 03 package [24]. After that, the finite field method is used to evaluate the longitudinal polarizability $\left(\alpha_{L}\right)$ and second hyperpolarizability $\left(\gamma_{L}\right)$ with the following numerical formulae:

$$
\begin{aligned}
& \alpha_{L}=-\frac{E(\delta F)+E(-\delta F)-2 E(0)}{(\delta F)^{2}}, \\
& \gamma_{L}=-\frac{E(2 \delta F)-4 E(\delta F)+6 E(0)-4 E(-\delta F)+E(-2 \delta F)}{(\delta F)^{4}} .
\end{aligned}
$$

The electric fields used are $0,8,16 \times 10^{-4}$ a.u. (i.e., $\delta F=$ $8 \times 10^{-4}$ a.u). As is known, large electric field will result in troubles in convergence and small electric field will require more significant figures for numerical derivatives and consequently is more computationally expensive. We found $\delta F=8 \times 10^{-4}$ a.u can work well for systems studied in this work. The electric field is applied along the direction of chain, as is chosen as $x$ axis direction. Only longitudinal components $\alpha_{x x}\left(\alpha_{L}\right)$ and $\gamma_{x x x x}\left(\gamma_{L}\right)$ are calculated.

\section{Results and Discussions}

The results of longitudinal polarizabilities per unit cell $\alpha_{L} / N$ ( $N$ is number of unit cells or double bonds in the system) are gathered in Table 1. And the data obtained with 6-31G basis set are plotted in Figure 1. Champagne et al. reported their calculations in [12] for $\mathrm{C}_{12} \mathrm{H}_{14}$ and $\mathrm{C}_{20} \mathrm{H}_{22}$ with same geometries (double zeta basis set used for $\mathrm{C}_{12} \mathrm{H}_{14}$ and 6-31G used for the other, see details in [12]). DFT data reported here for $\mathrm{C}_{20} \mathrm{H}_{22}$ are exactly the same as those in [12] (all data mentioned here and after can be found in Tables 1-4 in [12]) as expected. Small differences (SVWN: 94.8, B3LYP: 87.0, reported in [12]) for $\mathrm{C}_{12} \mathrm{H}_{14}$ can attribute to different basis set used in calculations. This can also explain the difference between HF and MP2 results; besides, that coupled HF (CHF) was used in [12]. In Figure 1, all curves obtained by DFT schemes are above HF; however, MP2 and CCSD curves are below that of HF. One can easily see the failure of commonly used xc-fuctionals here, as was shown before by others [12-14]. SVWN and BLYP predict nearly identical results. Another point worth to mention is the difference between CCSD and MP2 increases as the chain grows. It becomes more important while quantitative or semiquantitative results

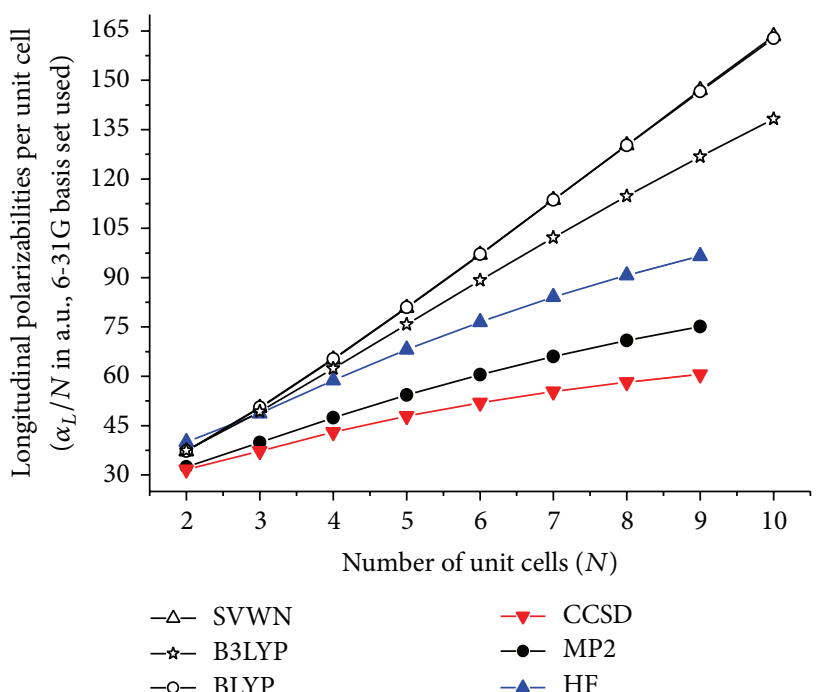

FIgURE 1: Longitudinal polarizabilities per unit cell $\left(\alpha_{L} / N\right)$ of polyenes (in a.u.) obtained by various quantum chemical methods with 6-31G basis set.

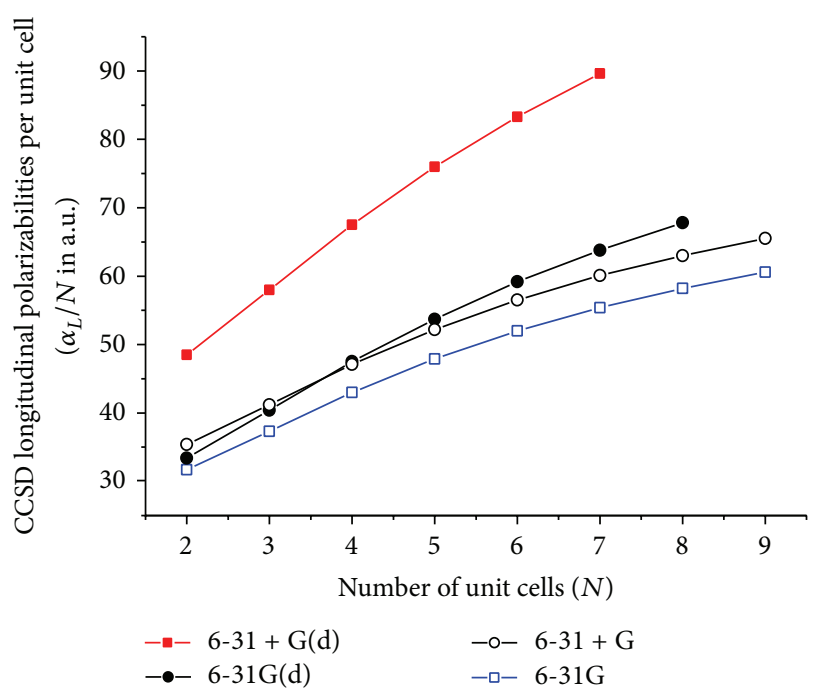

FIGURE 2: Longitudinal polarizabilities per unit cell $\left(\alpha_{L} / N\right)$ of polyenes (in a.u.) obtained by CCSD with various basis sets.

are required for long polyenes. Similar conclusions can be made for other basis sets from Table 1.

From Table 1, we conclude that basis set effects are unimportant for all DFT methods used here. Things are different for HF, MP2, and CCSD methods. We plot the CCSD results with various basis sets in Figure 2. The largest and also the most reliable basis set $6-31+G(d)$ is steeper than the other three ones. The differences between $6-31+G(d)$ basis set and other basis sets range from $27 \%$ to $38 \%$. There are no essential difference between 6-31G, 6-31+G, and 6-31G(d) basis sets.

We now come to $\gamma_{L}$. The results are listed in Table 2, and the data obtained with 6-31G basis set are plotted in Figure 3. We first discuss results for 6-31G basis set. For DFT methods with 6-31G basis set, negligible differences 
TABLE 1: Longitudinal polarizabilities per unit cell $\alpha_{L} / N$ of polyenes obtained by various chemical models (in a.u., $N$ is the number of unit cells or double bonds in the system).

\begin{tabular}{|c|c|c|c|c|c|c|c|c|c|}
\hline$N$ & 2 & 3 & 4 & 5 & 6 & 7 & 8 & 9 & 10 \\
\hline \multicolumn{10}{|l|}{$6-31 G$} \\
\hline HF & 40.0 & 48.7 & 58.8 & 68.1 & 76.5 & 84.1 & 90.7 & 96.6 & \\
\hline MP2 & 32.5 & 39.9 & 47.4 & 54.3 & 60.5 & 66.0 & 70.9 & 75.1 & \\
\hline CCSD & 31.7 & 37.3 & 43.0 & 47.9 & 52.0 & 55.4 & 58.2 & 60.6 & \\
\hline SVWN & 37.2 & 50.4 & 65.1 & 80.8 & 97.0 & 114 & 130 & 147 & 163 \\
\hline BLYP & 37.3 & 50.6 & 65.3 & 81.0 & 97.1 & 114 & 130 & 147 & 163 \\
\hline B3LYP & 37.4 & 49.3 & 62.4 & 75.8 & 89.2 & 102 & 115 & 127 & 138 \\
\hline \multicolumn{10}{|l|}{$6-31 G(d)$} \\
\hline HF & 39.4 & 48.4 & 58.4 & 67.7 & 76.1 & 83.5 & 90.1 & & \\
\hline MP2 & 33.5 & 42.0 & 50.6 & 58.7 & 66.1 & 72.8 & 78.7 & & \\
\hline CCSD & 33.4 & 40.4 & 47.5 & 53.7 & 59.2 & 63.8 & 67.8 & & \\
\hline SVWN & 37.4 & 51.1 & 66.0 & 82.0 & 98.6 & 116 & 133 & 150 & 167 \\
\hline BLYP & 37.5 & 51.2 & 66.1 & 82.0 & 98.5 & 115 & 132 & 149 & 166 \\
\hline B3LYP & 37.5 & 49.8 & 63.1 & 76.8 & 90.4 & 104 & 117 & 129 & 141 \\
\hline \multicolumn{10}{|l|}{$6-31+G$} \\
\hline HF & 43.7 & 52.6 & 62.8 & 72.2 & 80.7 & 88.3 & 94.9 & 101 & \\
\hline MP2 & 36.8 & 44.5 & 52.3 & 59.5 & 65.9 & 71.7 & 76.7 & 81.1 & \\
\hline CCSD & 35.4 & 41.2 & 47.1 & 52.2 & 56.5 & 60.1 & 63.0 & 65.5 & \\
\hline SVWN & 41.6 & 55.3 & 70.4 & 86.5 & 103 & 120 & 137 & 154 & 171 \\
\hline BLYP & 42.4 & 56.3 & 71.5 & 87.6 & 104 & 121 & 138 & 155 & 171 \\
\hline B3LYP & 41.8 & 54.2 & 67.6 & 81.3 & 94.9 & 108 & 121 & 133 & 145 \\
\hline \multicolumn{10}{|l|}{$6-31+G(d)$} \\
\hline HF & 56.4 & 68.4 & 81.8 & 94.1 & 105 & 115 & & & \\
\hline MP2 & 49.3 & 61.0 & 72.7 & 83.6 & 93.6 & 103 & & & \\
\hline CCSD & 48.5 & 58.0 & 67.5 & 76.0 & 83.3 & 89.6 & & & \\
\hline SVWN & 41.9 & 56.1 & 71.6 & 87.9 & 105 & 122 & 140 & 157 & 174 \\
\hline BLYP & 42.7 & 57.1 & 72.6 & 89.0 & 106 & 123 & 140 & 157 & 174 \\
\hline B3LYP & 42.1 & 54.9 & 68.6 & 82.6 & 96.5 & 110 & 123 & 135 & 147 \\
\hline
\end{tabular}

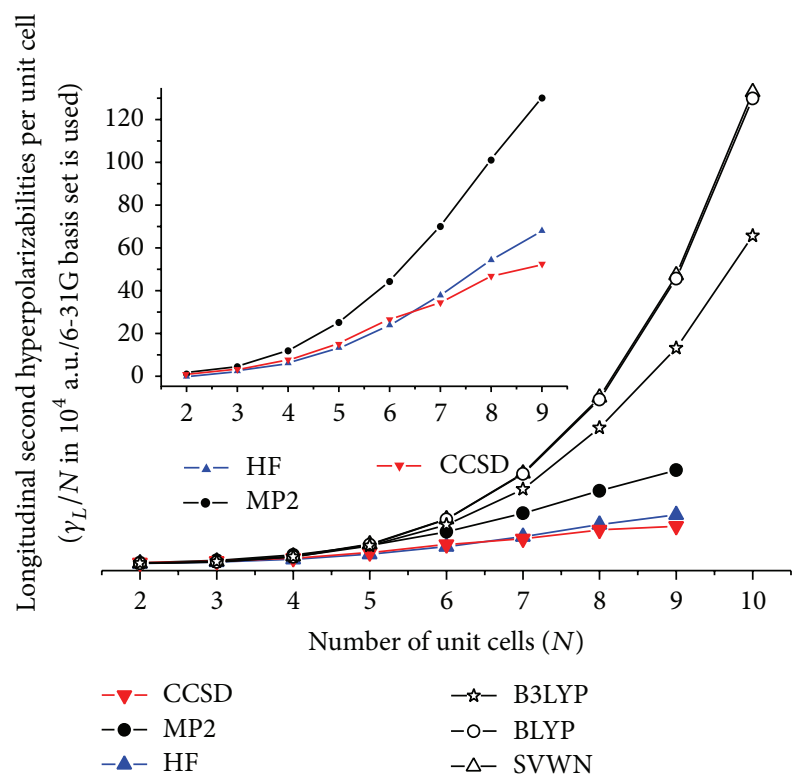

FIgURE 3: Longitudinal second hyperpolarizabilities per unit cell $\left(\gamma_{L} / N\right)$ of polyenes (in $10^{4}$ a.u.) obtained by various quantum chemical methods with 6-31G basis set. appear when compared to those reported in [12]. One more thing to be mentioned here is that electric fields and numerical derivatives formulae will result in some differences on digitals. It is very interesting, seen from Figure 3, that CCSD calculations predict electron correlation will reduce $\gamma_{L}$ while $N>6$, fully contrary to those predicted by MP2, but the question is whether this point can be also confirmed by more reliable basis sets. Similar results can be found for calculations with $6-31+G$ basis set and now the sign inversion of correlation contribution occurs at $\mathrm{C}_{18} \mathrm{H}_{20}$, while, for $6-31 G(d)$ and $6-31+G(d)$, this sign inversion has not been observed. One interesting problem then appears whether this sign inversion will occur or not for those two basis sets. If the answer is yes (we believe so), the commonly used MP2 (due to its moderate computational costs) makes qualitatively wrong predictions for correlation contribution on $\gamma_{L}$ for long polyenes. Undoubtedly, further work is necessary to completely confirm this. The local CCSD method developed recently may be helpful to investigate the longer polyenes [10, 25-32].

In Figure 4 , we plotted $\gamma_{L} / N$ calculated by CCSD with various basis sets. Again, $6-31+\mathrm{G}(\mathrm{d})$ basis set performs 
TABLE 2: Longitudinal second hyperpolarizabilities per unit cell $\gamma_{L} / N$ of polyenes obtained by various chemical models (in $10^{4}$ a.u., $N$ is the number of unit cells or double bonds in the system).

\begin{tabular}{|c|c|c|c|c|c|c|c|c|c|}
\hline$N$ & 2 & 3 & 4 & 5 & 6 & 7 & 8 & 9 & 10 \\
\hline \multicolumn{10}{|l|}{$6-31 G$} \\
\hline $\mathrm{HF}$ & -0.12 & 1.93 & 5.85 & 12.9 & 23.6 & 37.6 & 54.5 & 67.8 & \\
\hline MP2 & 0.84 & 4.33 & 11. 8 & 24.9 & 44.4 & 70.2 & 101 & 131 & \\
\hline CCSD & 0.80 & 3.11 & 7.44 & 15.3 & 26.5 & 34.5 & 46.7 & 52.3 & \\
\hline SVWN & 0.15 & 2.80 & 9.91 & 26.9 & 61.8 & 126 & 232 & 403 & 659 \\
\hline BLYP & 0.15 & 2.09 & 9.30 & 26.1 & 61.4 & 125 & 229 & 398 & 650 \\
\hline B3LYP & 0.14 & 2.29 & 9.25 & 24.5 & 54.2 & 104 & 190 & 301 & 458 \\
\hline \multicolumn{10}{|l|}{$6-31 G(d)$} \\
\hline $\mathrm{HF}$ & -0.11 & 1.79 & 5.38 & 11.9 & 21.4 & 33.9 & 49.3 & & \\
\hline MP2 & 0.67 & 3.83 & 10.8 & 23.4 & 42.2 & 67.4 & 98.8 & & \\
\hline CCSD & 0.60 & 4.04 & 8.71 & 16.6 & 27.2 & 40.3 & 56.3 & & \\
\hline SVWN & 0.14 & 2.59 & 9.26 & 22.6 & 63.9 & 116 & 232 & 392 & 644 \\
\hline BLYP & 0.13 & 2.57 & 9.25 & 23.1 & 60.0 & 121 & 231 & 384 & 637 \\
\hline B3LYP & 0.12 & 2.63 & 9.07 & 23.5 & 51.6 & 99.2 & 173 & 278 & 422 \\
\hline \multicolumn{10}{|l|}{$6-31+\mathrm{G}$} \\
\hline $\mathrm{HF}$ & 0.42 & 3.56 & 8.78 & 17.4 & 30.0 & 45.5 & 62.3 & 85.2 & \\
\hline MP2 & 1.92 & 7.08 & 16.9 & 33.0 & 56.1 & 85.6 & 125 & 161 & \\
\hline CCSD & 1.59 & 5.39 & 11.7 & 21.0 & 31.8 & 45.5 & 63.2 & 70.8 & \\
\hline SVWN & 1.07 & 5.45 & 15.4 & 37.2 & 79.3 & 158 & 285 & 433 & 711 \\
\hline BLYP & 1.18 & 5.71 & 16.9 & 40.0 & 83.2 & 159 & 357 & 468 & 728 \\
\hline B3LYP & 0.98 & 5.30 & 15.2 & 35.1 & 69.0 & 148 & 216 & 335 & 497 \\
\hline \multicolumn{10}{|l|}{$6-31+G(d)$} \\
\hline HF & 0.75 & 5.80 & 14.1 & 26.4 & 46.7 & 71.5 & & & \\
\hline MP2 & 2.88 & 11.1 & 26.8 & 51.5 & 91.5 & 141 & & & \\
\hline CCSD & 2.70 & 9.01 & 19.5 & 35.3 & 59.3 & 89.0 & & & \\
\hline SVWN & 1.08 & 5.26 & 14.76 & 35.5 & 76.0 & 148 & 269 & 450 & 709 \\
\hline BLYP & 1.19 & 5.76 & 15.2 & 36.1 & 78.8 & 154 & 280 & 468 & 782 \\
\hline B3LYP & 0.99 & 5.27 & 13.9 & 31.0 & 66.6 & 117 & 209 & 342 & 485 \\
\hline
\end{tabular}

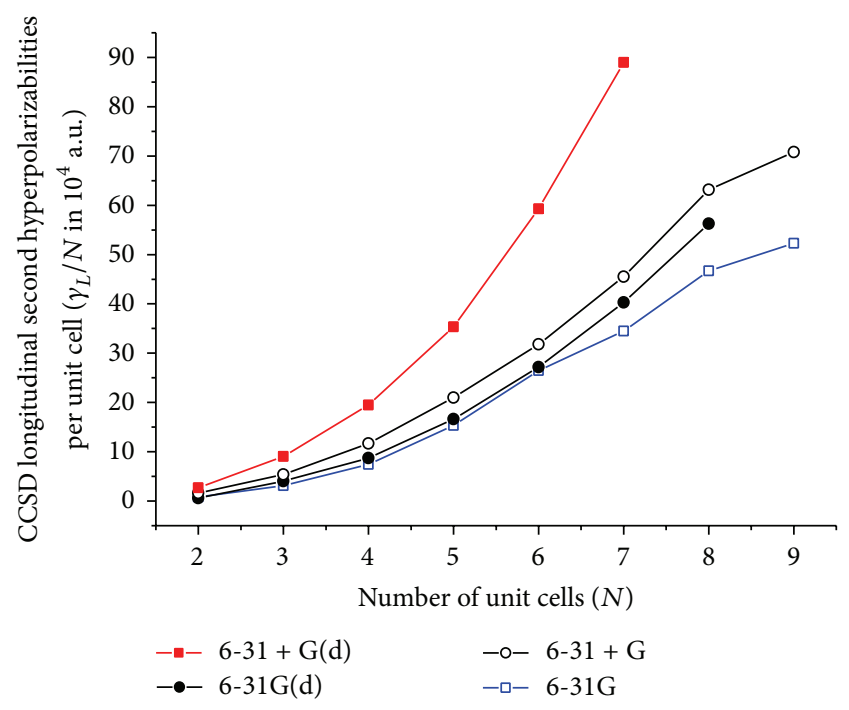

FIGURE 4: Longitudinal second hyperpolarizabilities per unit cell $\left(\gamma_{L} / N\right.$, in $10^{4}$ a.u.) obtained by CCSD with various basis sets. obviously different from the other three. The difference for $\gamma_{L} / N$ between $6-31+\mathrm{G}(\mathrm{d})$ basis set and other basis sets ranges from $41 \%$ to $61 \%$. And the $6-31 \mathrm{G}, 6-31+\mathrm{G}$, and $6-31 \mathrm{G}(\mathrm{d})$ basis sets perform similarly.

Sekino et al. reported their results based on CCSD calculations with cc-pVDZ basis set for polyenes up to $\mathrm{C}_{12} \mathrm{H}_{14}$ with a very different geometry (in the sense of bond length alternation) [15]. In their results, both CCSD and MP2 predict a positive correlation contribution for $\gamma_{L}$ up to $N=6$. Limacher et al. reported similar results based on calculations with response theory methods [33].

\section{Conclusions}

We have calculated the longitudinal polarizabilities and second hyperpolarizabilities of polyenes using HF, MP2, and CCSD methods and 6-31G, 6-31+G, 6-31G(d), and 6$31+\mathrm{G}(\mathrm{d})$ basis sets. Both MP2 and CCSD predict a negative correlation contribution for longitudinal polarizability, while DFT calculations using SVWN, BLYP, and B3LYP schemes all 
predicts positive correlation contributions. For second hyperpolarizability, a sign inversion of correlation contribution is observed for CCSD calculations with $6-31 \mathrm{G}$ and $6-31+\mathrm{G}$ basis sets. This sign inversion is yet to be further confirmed by more reliable basis set in the future. Contrary to CCSD, MP2 predicts a positive correlation contribution and thus should be used with much caution for evaluation of second hyperpolarizabilities of long conjugated chains. Addition of diffuse and polarization functions will enhance $\gamma_{L}$ obviously, but addition of polarization functions or addition of diffuse functions makes no essential difference.

\section{Conflict of Interests}

The authors declare that there is no conflict of interests regarding the publication of this paper.

\section{Acknowledgments}

This work is supported by National Natural Science Foundation of China (NSFC: 20903123), Natural Science Foundation Project of CQ CSTC (2010BB4116), and The Research Station Funding for Doctorial Faculty Members of Chongqing University of Posts and Telecommunications (A2008-16).

\section{References}

[1] G. Hurst, M. Dupuis, and E. Clementi, "Ab initio analytic polarizability, first and second hyperpolarizabilities of large conjugated organic molecules: applications to polyenes $\mathrm{C}_{4} \mathrm{H}_{6}$ to $\mathrm{C}_{22} \mathrm{H}_{24}$, The Journal of Chemical Physics, vol. 89, no. 1, pp. 385395,1988 .

[2] B. Kirtman and B. Champagne, "Nonlinear optical properties of quasilinear conjugated oligomers, polymers and organic molecules," International Reviews in Physical Chemistry, vol. 16, no. 4, pp. 389-420, 1997.

[3] B. M. Pierce, "A theoretical analysis of third-order nonlinear optical properties of linear polyenes and benzene," The Journal of Chemical Physics, vol. 91, no. 2, pp. 791-811, 1989.

[4] D. R. Kanis, M. A. Ratner, and T. J. Marks, "Third-order nonlinear optical response in organic materials: theoretical and experimental aspects," Chemical Reviews, vol. 94, no. 1, pp. 195242,1994

[5] C. Adant, J. L. Brédas, and M. Dupuis, "An ab initio and semiempirical study of the first- and third-order polarizabilities in benzene and thiophene derivatives: electron correlation effects," Journal of Physical Chemistry A, vol. 101, no. 16, pp. 3025-3031, 1997.

[6] D. Jacquemin, J. M. André, and E. A. Perpète, "Geometry, dipole moment, polarizability and first hyperpolarizability of polymethineimlne: an assessment of electron correlation contributions," Journal of Chemical Physics, vol. 121, no. 9, pp. 43894396, 2004.

[7] D. Jacquemin, E. A. Perpète, and J. André, "NLO response of polymethineimine and polymethineimine/polyacetylene conformers: assessment of electron correlation effects," International Journal of Quantum Chemistry, vol. 105, no. 6, pp. 553$563,2005$.
[8] H. Sekino and R. J. Bartlett, "Hyperpolarizabilities of molecules with frequency dependence and electron correlation," The Journal of Chemical Physics, vol. 94, no. 5, pp. 3665-3670, 1991.

[9] Q. X. Li, L. P. Chen, and Z. G. Shuai, "Electron correlation effects on the nonlinear optical properties of conjugated polyenes," Chemical Physics Letters, vol. 457, no. 1-3, pp. 276-278, 2008.

[10] Q. X. Li, Y. P. Yi, and Z. G. Shuai, "Local approach to coupled cluster evaluation of polarizabilities for long conjugated molecules," Journal of Computational Chemistry, vol. 29, no. 10, pp. 1650-1655, 2008.

[11] M. Torrent-Sucarrat, M. Solà, M. Duran, J. M. Luis, and B. Kirtman, "Basis set and electron correlation effects on ab initio electronic and vibrational nonlinear optical properties of conjugated organic molecules," Journal of Chemical Physics, vol. 118, no. 2, pp. 711-718, 2003.

[12] B. Champagne, E. A. Perpète, S. J. A. Van Gisbergen et al., "Assessment of conventional density functional schemes for computing the polarizabilities and hyperpolarizabilities of conjugated oligomers: an ab initio investigation of polyacetylene chains," Journal of Chemical Physics, vol. 109, no. 23, pp. 1048910498, 1998.

[13] S. J. A. Van Gisbergen, P. R. T. Schipper, O. V. Gritsenko et al., "Electric field dependence of the exchange-correlation potential in molecular chains," Physical Review Letters, vol. 83, no. 4, pp. 694-697, 1999.

[14] B. Champagne, E. A. Perpète, D. Jacquemin et al., "Assessment of conventional density functional schemes for computing the dipole moment and (hyper)polarizabilities of push-pull $\pi$ conjugated systems," Journal of Physical Chemistry A, vol. 104, no. 20, pp. 4755-4763, 2000.

[15] H. Sekino, Y. Maeda, M. Kamiya, and K. Hirao, "Polarizability and second hyperpolarizability evaluation of long molecules by the density functional theory with long-range correction," Journal of Chemical Physics, vol. 126, no. 1, Article ID 014107, 6 pages, 2007.

[16] Z. G. Shuai and J. L. Brédas, "Coupled-cluster approach for studying the electronic and nonlinear optical properties of conjugated molecules," Physical Review B, vol. 62, no. 23, pp. 15452-15460, 2000.

[17] H. Sekino and R. J. Bartlett, "Molecular hyperpolarizabilities," The Journal of Chemical Physics, vol. 98, no. 4, pp. 3022-3037, 1993.

[18] G. Maroulis, "A systematic study of basis set, electron correlation, and geometry effects on the electric multipole moments, polarizability, and hyperpolarizability of HCI," The Journal of Chemical Physics, vol. 108, no. 13, pp. 5432-5448, 1998.

[19] G. Maroulis, "On the accurate theoretical determination of the static hyperpolarizability of trans-butadiene," The Journal of Chemical Physics, vol. 111, no. 2, pp. 583-591, 1999.

[20] S. Nénon, B. Champagne, and M. I. Spassova, "Assessing longrange corrected functionals with physically-adjusted rangeseparated parameters for calculating the polarizability and the second hyperpolarizability of polydiacetylene and polybutatriene chains," Physical Chemistry Chemical Physics, vol. 16, no. 15, pp. 7083-7088, 2014.

[21] M. de Wergifosse and B. Champagne, "Electron correlation effects on the first hyperpolarizability of push-pull- $\pi$ conjugated systems," The Journal of Chemical Physics, vol. 134, no. 7, Article ID 074113, 2011.

[22] R. G. Parr and W. T. Yang, Density-Functional Theory of Atoms and Molecules, Oxford University Press, New York, NY, USA, 1989. 
[23] W. Koch and M. C. Holthausen, A Chemist's Guide to Density Functional Theory, Wiley-VCH, New York, NY, USA, 2000.

[24] C. Revision, M. J. Frisch, G. W. Trucks et al., "Gaussian 03, Revision C.02," Gaussian, Wallingford, Conn, USA, 2004.

[25] C. Hampel and H. Werner, "Local treatment of electron correlation in coupled cluster theory," Journal of Chemical Physics, vol. 104, no. 16, pp. 6286-6297, 1996.

[26] M. Schütz and H. Werner, "Low-order scaling local electron correlation methods. IV. Linear scaling local coupled-cluster (LCCSD)," The Journal of Chemical Physics, vol. 114, no. 2, pp. 661-681, 2001.

[27] M. Schütz and H. Werner, "Local perturbative triples correction (T) with linear cost scaling," Chemical Physics Letters, vol. 318, no. 4-5, pp. 370-378, 2000.

[28] S. H. Li, J. Ma, and Y. S. Jiang, "Linear scaling local correlation approach for solving the coupled cluster equations of large systems," Journal of Computational Chemistry, vol. 23, no. 2, pp. 237-244, 2002.

[29] S. H. Li, J. Shen, W. Li, and Y. Jiang, "An efficient implementation of the "cluster-in-molecule" approach for local electron correlation calculations," The Journal of Chemical Physics, vol. 125, no. 7, Article ID 074109, 10 pages, 2006.

[30] N. Flocke and R. J. Bartlett, "Localized correlation treatment using natural bond orbitals," Chemical Physics Letters, vol. 367, no. 1-2, pp. 80-89, 2003.

[31] N. Flocke and R. J. Bartlett, "A natural linear scaling coupledcluster method," Journal of Chemical Physics, vol. 121, no. 22, article no. 10, pp. 10935-10944, 2004.

[32] G. E. Scuseria and P. Y. Ayala, "Linear scaling coupled cluster and perturbation theories in the atomic orbital basis," The Journal of Chemical Physics, vol. 111, no. 18, pp. 8330-8343, 1999.

[33] P. A. Limacher, Q. X. Li, and H. P. Lüthi, "On the effect of electron correlation on the static second hyperpolarizability of conjugated oligomer chains," Journal of Chemical Physics, vol. 135, no. 1, Article ID 014111, pp. 1-4, 2011. 

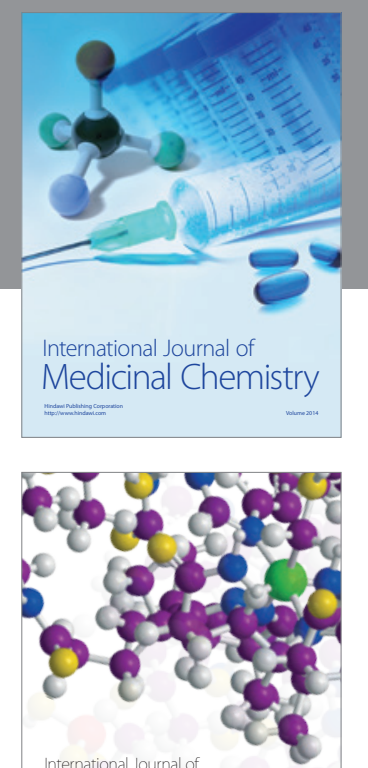

\section{Carbohydrate} Chemistry

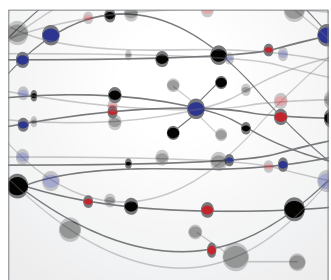

The Scientific World Journal
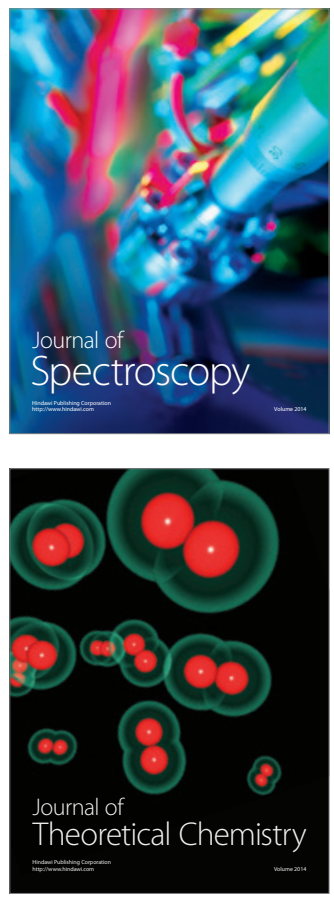
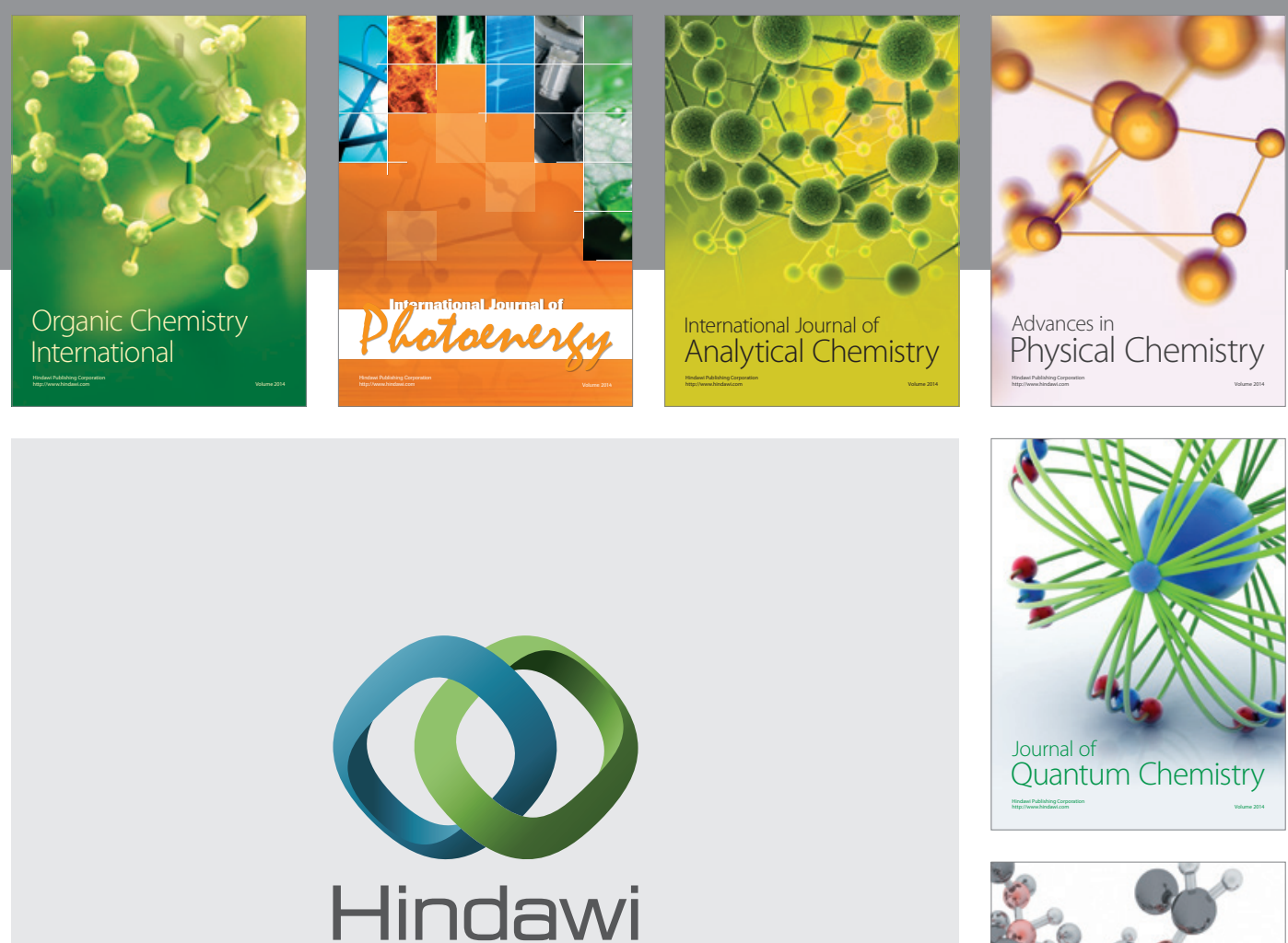

Submit your manuscripts at

http://www.hindawi.com

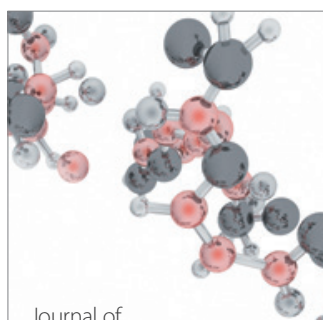

Analytical Methods

in Chemistry

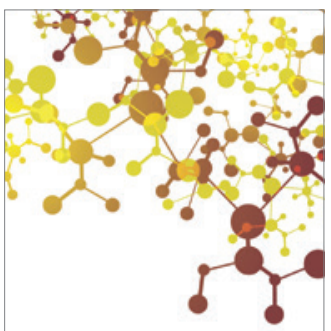

Journal of

Applied Chemistry

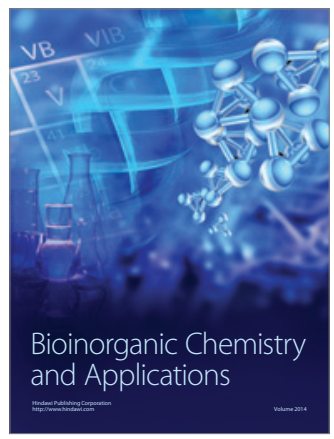

Inorganic Chemistry
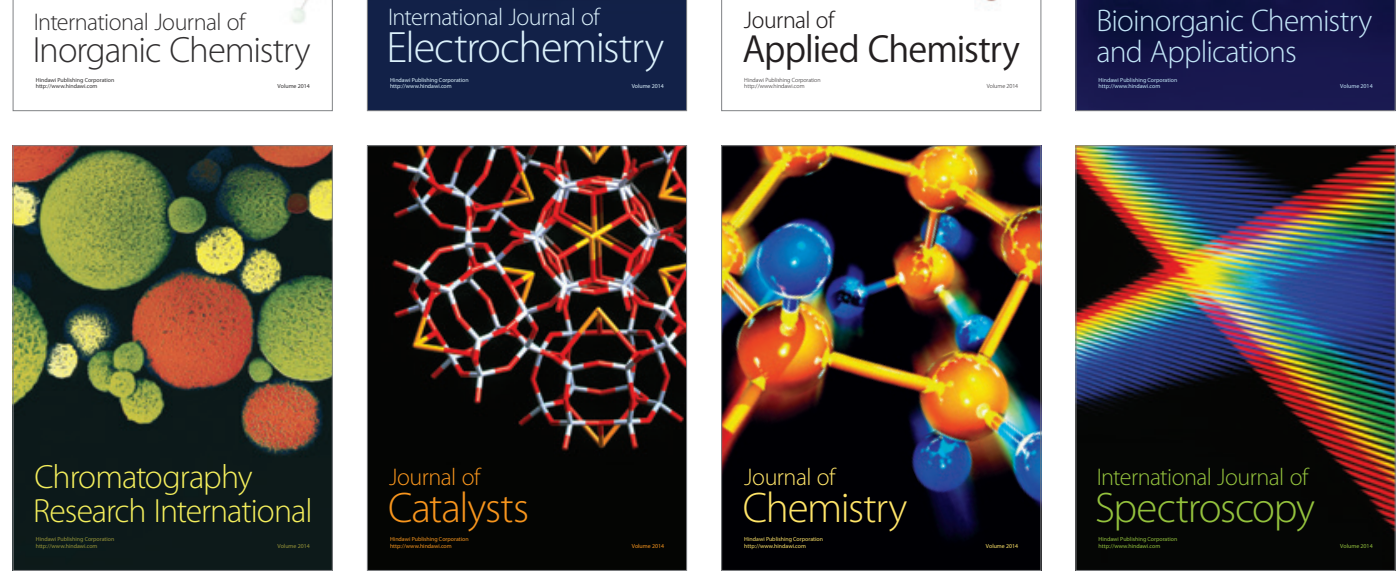